\title{
Research on Intelligent Detecting Technology Based on Extension Fuzzy Method
}

\author{
Wang Minghui, Yu Yongquan \\ Faculty of Computer Science \\ Guangdong University of Technology \\ Guangzhou, China \\ wflmh@21cn.com
}

\author{
Wei Ge, Ren Xiang, Liu Dan, Wei Yujing \\ Guangdong Jin Fu information technology co., LTD \\ Foshan, China \\ 411213786@qq.com
}

\begin{abstract}
This paper studies a new kind of intelligent detecting technology which includes key detectable matterelements distilling, adaptive degree analyzes, focusing and reasoning mechanism construction, focusing processing. This paper researches on the focusing and reasoning mechanism construction, which is to construct the reasoning mechanism and use it to get the focusing. The focusing is actually to work out the core element of the detectable matter-element according to the adaptive degree distributing. This paper puts forward the methods to solve all types of focusing: to the one-dimensional and two-dimensional focusing, connects the adaptive degree with the dependent function to figure out the focusing; to the threedimensional and multidimensional focusing, constructs an extension fuzzy -based knowledge reasoning expert system and uses the extension reasoning and fuzzy rules to work out the focus.
\end{abstract}

Keywords-Intelligent detecting; Extension method; Fuzzy rule; Elements focusing; Extension reasoning

\section{INTRODUCTION}

With the rapid development of computer science and information technology, the detection technology has become the key technology for the economic development and scientific progress. For the detection technical flaws, it is inevitable that many detections bog down. To solve the undetectable problems, the extension detecting technology[1] emerges as the times require. It uses the theoretical foundation and the analytical method of contradiction as well as the relatively mature sensor technology to make the undetectable problem detectable.

Information detection is one of the current research topics of information subject and plays an important role in development. Although kinds of information detection technology are developing increasingly, it has solved the problem which in the examination domain exists to some extent. Two problems, how to detect undetectable thing and how to improve the detecting accuracy, still could not be solved well. Extecnis [2-3]is based on the extension of matter. It establishes a set of formal method and solves the contradictory problem in data-based way and is widely used in many fields[4-6]. Extension detection based on extenics solves how to detect the character and value of non-detectable matter-element by transforming the detectable matter-element and solve detecting every kind of information which cannot to be detected or is difficult to be detected.

\section{Focus Visualization MEChanism ModeL}

\section{A. Focus Result Analysis}

As to one same matter and due to the characteristic of multifeature, the un-measurable matter-element is often hidden by the measurable characteristics of the same matter. The unmeasurable matter-element can obtained from the measurable matter-element through the extension transformation. The influences of accuracy, stability and fault tolerance of transformation relationship make the detection result has three typical results: the dispersion of quantity value field, the dispersion of quantity value line and the dispersion of quantity value point. The matter-element focus is the way that using the extension reasoning of knowledge, calculating its reasonable key element according to the elements distribution space of detection result of several measurable matter-elements which is obtain the corresponding focus by filtering the interfering noise.

The un-measurable matter-element can obtained through the extension transformation of one measurable matter-element but also the extension transformation of two and more measurable matter-elements. If the quantity of measurable matter-elements is different, so as the focusing dimensionality of focus. Correspondingly, the one-dimension focus means there is one measurable matter-element is influencing the targeted un-measurable matter-element, the two-dimension focus means there are two measurable matter-elements are influencing the targeted un-measurable matter-element, and by that analogy, the n-dimension focus means there are $n$ measurable matter-elements are influencing the targeted unmeasurable matter-element.

The focus visualization of measurable matter-element is essentially the process of evaluate the element focusing result and actual un-measurable matter-element to obtain the detection reliability. The focus produced through the elements focusing could be one smaller surface or multi-surface, so the visualization treatment is required to obtain the optimal result. This optimal result is the comprehensive detection result of multi measurable matter-element that truly wants to gained. Focus Visualization Mechanism Model 
The focus is the filtering output of measurable matterelement detection result, it just narrowed the range of consideration to a certain range and did not obtain the final detection result. The focus visualization mechanism is on the basis of the focus result and preliminary extension analysis to further determine the focus, make the optimal decision and evaluate the extension detection scheme to complete the detection mission of un-measurable matter-element.

The followings are the introductions of relevant concepts of each module in the model:

Focus Information Database: the set of measurable matterelement focus. The Focus Information Database includes each focus information and its focus element information.

Focus Element: The composition elements of focus. If the focus is one point, then the focus only has one focus element; if the focus is one surface or multi-surface, then the focus includes multi focus elements. One focus element includes $n(n \geq 1)$ of un-measurable matter-element.

Bi-directional Transformation of Matter-element: The normal and inverse transformation that carried out between the measurable and un-measurable matter-elements which combined the fuzzy rule.

Normal Transformation: The transformation that from unmeasurable matter-element into measurable matter-element which can be used to determine the transformation result of multi measurable matter-elements.

Inverse Transformation: The transformation that from measurable matter-element into un-measurable matter-element which can be used to determine the focus situation of multi measurable matter-elements detection focusing.

Source Matter-element: The input matter-element in the bidirectional transformation, the source matter-element is the un-measurable matter-element in the normal transformation and the measurable matter-element in the inverse transformation.

Targeted Matter-element: The matter-element whose transformation value is obtained through the bi-directional transformation, it is usually used as a reference index and transformation output to make a comparison, the targeted matter-element is the measurable matter-element in the normal transformation and the un-measurable matter-element in the inverse transformation.

Transformed Matter-element: The matter-element used as the output of bi-directional transformation, it could be the measurable matter-element or un-measurable matter-element according to the different directions of transformation.

Extension Transformation Database: The set of extension transformation that carried out between the measurable and un-measurable matter-elements which can be organized with the extension inference tree.

Pretreatment: Combining the extension transformation to inspect the extension inference link between the measurable and un-measurable matter-elements and determine the quality index of focus.

Fuzzy Rule Database: The fuzzy inference rule set of the measurable and un-measurable matter-elements.

Measure Model: The evaluation model that represented by the range value of detection and the relationship characteristic value or characteristic function of un-measurable and measurable matter-element. The error can be calculated out through the comparison between the targeted and transformed matter-elements, the information such as error can be used to measure the focus.

Visualization Result: According to the precision and measure function given by the measure model, the require-tomeasure result of the transformation from measurable matterelement to un-measurable matter-element can be obtained by the means of determination of focus elements.

\section{Focus Visualization InfERENCE MEChanism}

The bi-directional determination of measurable matterelement and un-measurable matter-element is required by the visualization treatment and the bi-directional determination takes the bi-directional transformation between both of them as its basis. The normal transformation that from the unmeasurable matter-element to measurable matter-element is used to determine the transformation result of multi measurable matter-element; the inverse transformation that from the measurable matter-element to un-measurable matterelement is used to determine the focus situation of multi measurable matter-element focusing. Due to the complicated relationship between objective matters, the relationship between the measurable and un-measurable matter-element matter-elements is hard to represent with traditional mathematical formula. So the fuzzy inference is introduced to realize the bi-directional transformation between measurable matter-element and un-measurable matter-element. The fuzzy inference is based on the fuzziness of human thinking and there is essential difference between the fuzzy inference and traditional inference. The core of fuzzy inference is that it uses the linguistical condition statement with fuzziness as the inference rule; it uses the experiences of human expert to conduct inference in essence and fully reflects the intellectual activities of human being. So the fuzzy intellectual activity is required to realize the bi-directional transformation between measurable matter-element and un-measurable matterelements between measurable matter-element and unmeasurable matter-elements.

The inference is a thinking process which can obtain a new conclusion on the basis of one or multi known conclusion(s). Its structure includes two parts of premise and conclusion, the known conclusion is called as the premise of inference and the newly conclusion is called as the conclusion of inference. The correct inference requires true premise, it also requires to apply the inference pattern with formal logic conformance and obey the inference rules. The inference rule is the correct inference pattern, obey these patterns can guarantee the inference process is logical. Bi-directional Transformation Schematic Diagram is shown as fig.1.

The Superiority Evaluation Method is the basic method to evaluate the superiority and inferiority of one subject including matter, strategy and method. 


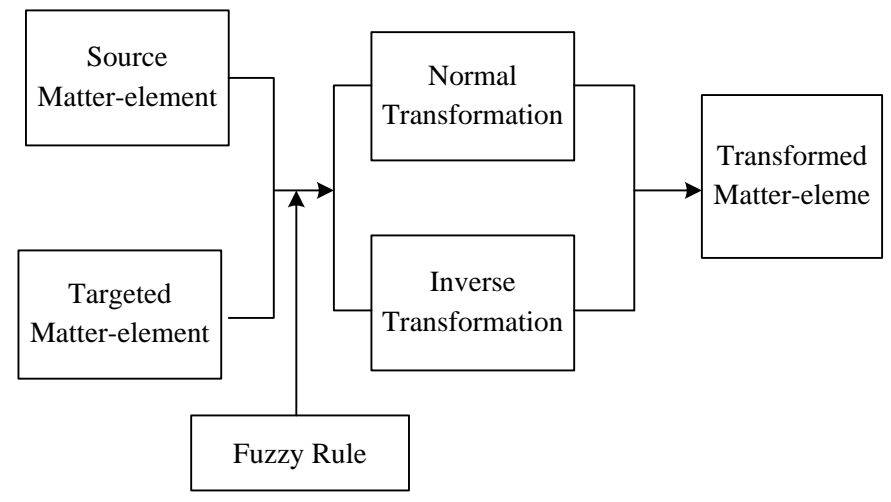

Fig .1. Bi-directional Transformation Schematic Diagram

As to the object-being-evaluated $\mathrm{Z}$, if the index to measure to the superiority and inferiority is $M$, the quantitative range that meet the requirement is $\mathrm{X} 0$, the value range allowed by the quantity value is $\mathrm{X}$, then take the $\mathrm{X} 0$ as classical field to establish the correlation function $\mathrm{K}(\mathrm{z})$ which represent the meet-the-requirement degree of object and call it as the correlation degree of $\mathrm{Z}$ about the measure condition $\mathrm{M}$.

In order to facilitate to compare the superiority and inferiority of solution, normalize the correlation degree firstly.

Normalization of Correlation Degree. If the correlation degree of the solution I of one specific question about the measure condition $\mathrm{M}$ is $\mathrm{K}(\mathrm{I})$, then:

$$
k_{I}=\left\{\begin{array}{lr}
\frac{K(I)}{\max _{x \in X_{0}} K(x)}, & K(I)>0 \\
\frac{K(I)}{\max _{x \notin X_{0}}|K(x)|}, & K(I)<0
\end{array}\right.
$$
M.

Call the solution I as the normalized correlation degree of

As to the object-being-evaluated $\mathrm{Z}$, if the measuring index set is $M=\left\{M_{1}, M_{2}, \cdots, M_{n}\right\}$, the normalized correlation degree of solution I about $M_{i}$ is $k_{i}(i=1,2, \cdots, n)$, the weight coefficient of $M_{i}$ is $\alpha_{i}$ (which indicates the real number of relative importance of this measuring index) $(i=1,2, \cdots, n)$, then the comprehensive correlation degree of all the measuring index is

$$
C(I)=\sum_{i=1}^{n} \alpha_{i} k_{i}
$$

Call it as the superiority of solution I.

Generally speaking, we assume when $C(I)>0$, it indicates the superiority is more than inferiority of this solution to this question; when $C(I)<0$, it indicates the inferiority is more than superiority of this solution to this question.

Concrete Procedure of Superiority Evaluation Method.

1st Step: In order to evaluate the superiority and inferiority of one subject, the measuring index must be stipulated firstly. The superiority and inferiority is just relative to one specific standard. As to one object, it is may superiority to some measuring index but also may inferiority to another measuring index. So the degree of superiority and inferiority and their possible changing situation must be reflected in the evaluation of the superiority and inferiority of one subject. So the measuring index $M=\left\{M_{1}, M_{2}, \cdots, M_{n}\right\}$ must be determined according to specific questions. Among them, the $M_{i}=\left(c_{i}, V_{i}\right)$ is the characteristic element, $c_{i}$ is the evaluating characteristic, $\quad V_{i}$ is the field value of quantification $(i=1,2, \cdots, n)$.

2nd Step: Weight Coefficient Determination. Each measuring index $M_{1}, M_{2}, M_{3}, \cdots, M_{n}$ to evaluate the superiority and inferiority of one subject $N_{j}(j)(j=1,2, \cdots, m) N_{j}(\mathrm{j}=1,2, \cdots, \mathrm{m})$ has the various weights, so using the weight coefficient to indicate the importance of each measuring condition. Use the index $\Lambda$ to indicate the must-be-satisfied conditions and as to other measuring conditions, assign different value in $[0,1]$ to each condition according to their importance, then the weight coefficient is:

$$
\alpha=\left(\alpha_{1}, \alpha_{2}, \cdots, \alpha_{n}\right)
$$

Among them, if $\alpha_{i_{0}}=\Lambda$, then $\sum_{\substack{k=1 \\ k \neq i_{0}}}^{n} \alpha_{k}=1$.

The size of weight coefficient plays a significant role in the evaluation of superiority, as different weight coefficients will produce different conclusions. But the weight coefficient is often determined by human which always has subjectivity and arbitrariness that will affect the evaluation authenticity and reliability of solution. We use the analytic hierarchy process to determine the order of importance of each measuring index, so as to determine the weight coefficient.

3rd Step: First Evaluation. Once the weight coefficient of each measuring condition has been determined, filter the mustbe-satisfied conditions and remove the objects did not meet the condition, process the following steps with the object that has met the must-be-satisfied condition $\Lambda$. (Assume all of $N_{1}, N_{2}, \cdots, N_{m}$ have met must-be-satisfied condition.)

4th Step: Establish the correlation function and calculate the correlation degree.

If the measuring index set is

$$
M=\left\{M_{1}, M_{2}, M_{3}, \cdots, M_{n}\right\}
$$

$M_{i}=\left(c_{i}, V_{i}\right)(i=1,2, \cdots, n)$, the weight coefficient assignment is $\alpha=\left(\alpha_{1}, \alpha_{2}, \cdots, \alpha_{n}\right)$.

Then establish the correlation function about $V_{1}, V_{2}, \cdots, V_{n}$ :

1) If $V_{i}$ is one interval $X_{0_{i}}$, then take the simple correlation function:

$$
K_{i}(x)=\frac{\rho\left(x, X_{0_{i}}\right)}{\left|X_{0_{i}}\right|} \quad(i=1,2, \cdots, n)
$$

2) If $V_{i}$ is a set of some discrete data, such as $M_{i}$ indicates the quality grade of product and $\mathrm{Vi}=\{\mathrm{A}$ grade, $\mathrm{B}$ grade, $\mathrm{C}$ grade $\}$, then take the simple correlation function

$$
K_{i}(x)= \begin{cases}a, & x=\text { Agrade } \\ b, & x=\text { Bgrade } \\ c, & x=\text { Cgrade }\end{cases}
$$


The values of $a, b, c$ can be scored and determined according to the expertise or historical data.

3) If $V_{i}$ is represented by the nested intervals without public point that constituted by $X_{0_{i}}$ and $X_{i}\left(X_{0_{i}} \subset X_{i}\right)$ and the optimal point of this measuring index is the midpoint of $X_{0_{i}}$, then the elementary correlation function can be taken as

$$
K_{i}\left(x_{i}\right)=\frac{\rho\left(x_{i}, X_{0_{i}}\right)}{\rho\left(x_{i}, X_{i}\right)-\rho\left(x_{i}, X_{0_{i}}\right)} \quad(i=1,2, \cdots, n)
$$

Denote the correlation function value of object $N_{j}$ about each measuring index $M_{i}$ as $K_{i}\left(N_{j}\right)$, then the correlation degree of each object $N_{1}, N_{2}, \cdots, N_{m}$ about $M_{i}$ is:

$$
K_{i}=\left(K_{i}\left(N_{1}\right), K_{i}\left(N_{2}\right), \cdots, K_{i}\left(N_{m}\right)\right), \quad i=1,2, \cdots, n
$$

5th Step: Normalization

$$
k_{i j}=\frac{K_{i}\left(N_{j}\right)}{\max \left|K_{i}(x)\right|}, \quad i=1,2, \cdots, n, j=1,2, \cdots, m,
$$

Then the normalizated correlation degree of each object $N_{1}, N_{2}, \cdots, N_{m}$ about $M_{i}$ is:

$$
k_{i}=\left(k_{i 1}, k_{i 2}, \cdots, k_{i m}\right), \quad i=1,2, \cdots, n
$$

\section{6th Step: Superiority Calculation}

If the normalizated correlation degree of object $N_{j}$ about each measuring index $M_{1}, M_{2}, M_{3}, \cdots, M_{n}$ is:

$$
\mathbf{K}\left(N_{j}\right)=\left[\begin{array}{l}
k_{1 j} \\
k_{2 j} \\
\vdots \\
k_{n j}
\end{array}\right], \quad j=1,2, \cdots, m
$$

Then take the superiority of $N_{j}$ as:

$$
C\left(N_{j}\right)=\alpha \mathbf{K}\left(N_{j}\right)=\left(\alpha_{1}, \alpha_{2}, \cdots, \alpha_{n}\right)\left[\begin{array}{l}
k_{1 j} \\
k_{2 j} \\
\vdots \\
k_{n j}
\end{array}\right]=\sum_{i=1}^{n} \alpha_{i} k_{i j}, j=1,2, \cdots, m
$$

Compare the superiority of , if $C\left(N_{0}\right)=\max _{j \in\{1,2, \cdots, m\}}\left\{C\left(N_{j}\right)\right\}$, then the object $N_{0}$ has higher superiority.

During the process of solving practical problems, some conditions are the must-be-satisfied, if the condition cannot be met, then the other conditions will not allow to use even them are again good. As to the object evaluation, one must not only consider the advantage aspect but also the disadvantage aspect, the applicable filtered scheme can be finally achieved by considering both aspects and conducting comprehensive evaluation. In addition, the dynamic nature must be considered during the evaluation to consider the potential advantages and disadvantages.

The superiority evaluation is proposed on the basis of these practical backgrounds and it has the following three steps:

1) Use to represent the must-be-satisfied conditions.
2) The value of correlation function could be positive or negative, so the superiority could reflect the degree of superiority and inferiority of one specific object.

3) Because the extension set could represent the variability, therefore it can measure the superiority and inferiority of one specific object from the development perspective after the parameter thas been introduced.

\section{CONCLUSIONS}

During the focus visualization process, the measurable and un-measurable matter-elements that consisted the focus and their extension inference procedure must be confirmed; the extension inference procedure must be analyzed so that the evaluation index of this extension inference procedure can be obtained and pretreated. In order to conduct comprehensive inspection of the extension transformation result and the focus situation, the model needs to conduct the bi-directional transformation to evaluate the focus element in the focus, because the relationship between the measurable and unmeasurable matter-elements usually is not the clear mathematical relationship, the bi-directional transformation is required to adopt the fuzzy inference which can process the problem of complexity, fuzziness and uncertainty. The measure model is used to evaluate the result of bi-directional transformation of focus element, when the error of transformation result is less than the given value of measure model, then the focus element is deemed to as reasonable, the un-measurable matter-element detection value of optimal focus element can be used as the final detection result.

\section{Acknowledgment}

The authors are grateful to Guangdong Provincial Natural Science Foundation of China(2013B090600028) for decisive support.

\section{References}

[1] Y.H. Chen, Y.Q.Yu,B.Zeng, "Extension detecting technology and the implementing method for its application in engineering," International Conference on Machine Learning and Cybernetics, 2009,pp.631- 636.

[2] Y.Q.Yu, Y Huang, M.H.Wang, The related matter-elements in extension detecting and application, International Conference on Information Technology and Applications, 2005, pp.411 - 414.

[3] X.Y Zhu, Y.Q Yu, Intrusion Detection System Model Based on ExtensionDetecting ,International Conference on Convergence Information Technology, 2007,pp.1536 - 1540.

[4] H.Q. Chen,Y.Q.Yu, Z.B.Chen, Automatically Compute The Classical Filed of Matter-Element Model Based on Genetic Algorithms.International Conference on Information Technology and Applications, 2005,pp.46-52.

[5] F.Ye, Y.Q.Yu,Automatic Fuzzy Rules Extraction of Generalized Fuzzy RBF Neural Network Based on Hierarchical Evolutionary Programming.The International Conference 2007 on Information Computing and Automation. 2007,pp.98-105.

[6] X.Y.Zhang,Y.Q. Yu An Extension-based Dynamic Load Balancing Model of Heterogeneous Server Cluster, IEEE International Conference on Granular Computing, 2007,pp.121-128. 